\title{
Caracterización de los factores de riesgo de diabetes mellitus tipo 2 mediante el test de Findrisk en una población de 30 a 50 años de Medellín, Colombia
}

\author{
Characterization of risk factors for type 2 diabetes mellitus by Findrisk \\ test in a population of 30 to 50 years old from Medellin, Colombia
}

\section{Shirley Montes-Ochoa MD', Karen Serna-Arrieta MD², Stephanie Estrada-Ávila $M D^{1}$, Foris R. Guerra-López $M D^{3}$, Isaura P. Sánchez PhD ${ }^{4}$}

Introducción: la diabetes mellitus es una de las enfermedades crónicas prevenibles con mayor impacto en la población mundial. Objetivo: caracterizar los factores de riesgo de diabetes mellitus tipo 2 mediante la aplicación del test de Findrisk. Materiales y métodos: se realizó un estudio piloto descriptivo que incluyó 51 personas entre 30 y 50 años, residentes en Medellín, Colombia, sin diagnóstico previo de diabetes mellitus. Se aplicó el test de Findrisk para valorar los factores de riesgo asociados a diabetes.

Resultados: el 68,6\% de las personas fueron mujeres. El 64,7\% de la población fue menor de 45 años. El 51,0\% de la población obtuvo puntaje menor que 7 (bajo), el 17,6\% entre 7 y 11 (elevado levemente), el 21,6\% entre 12 y 14 (moderado), el 7,8\% mayor que 14 y hasta 20 (alto) y el 2,0\% mayor que 20 (muy alto). El promedio del puntaje total fue 7,8. El 51,0\% de la población tuvo IMC menor que $25 \mathrm{Kg} / \mathrm{m}^{2}$, el 66,7\% no realizaba actividad física diaria, el 47,1\% no consumía frutas ni verduras, el 94,1\% no tenía antecedentes de niveles de glucosa

\footnotetext{
${ }^{1}$ Candidata a título de Médica, Facultad de Ciencias de la Salud, Programa de Medicina, Corporación Universitaria Remington. Medellín, Colombia

${ }^{2}$ Candidata a título de Médica, Facultad de Ciencias de la Salud, Programa de Medicina, Corporación Universitaria Remington. Medellín, Colombia. Correo electrónico: karen12serna@hotmail.com

${ }_{3}^{3}$ Médico, Internista. Hospital San Cristóbal. Magdalena, Colombia.

${ }^{4}$ Bióloga, PhD en Biología. Docente-Investigador, Grupo de Investigaciones Biomédicas UniRemington, Facultad de Ciencias de la Salud, Programa de Medicina, Corporación Universitaria Remington. Medellín, Colombia.

Conflicto de intereses: los autores declaran que no tienen conflicto de intereses

Medicina \& Laboratorio 2016; 22: 563-576

Módulo 19 (Investigación), número 50. Editora Médica Colombiana S.A. $2016^{\circ}$

Recibido el 31 de mayo de 2016; aceptado el 24 de octubre de 2016
} 
altos en sangre y el 23,5\% presentaba antecedentes familiares de diabetes mellitus en primer grado de consanguinidad. En el análisis de asociación la edad, el consumo de medicamentos antihipertensivos y los antecedentes familiares de diabetes mellitus tuvieron influencia significativa en el puntaje final del test. Conclusiones: el test de Findrisk es una herramienta útil para caracterizar los factores de riesgo de diabetes mellitus tipo 2.

Palabras claves: diabetes mellitus, intolerancia a la glucosa, enfermedades metabólicas, obesidad, test Findrisk.

Introduction: Diabetes mellitus is one of the preventable chronic diseases with greatest impact on global population. Objective: To characterize the risk factors for type 2 diabetes mellitus through the application of the Finnish Diabetes Risk Score. Materials and methods: A descriptive pilot study including 51 individuals between 30 and 50 years old, residing in Medellin, Colombia, without a previous diagnosis of diabetes mellitus was performed. The Finnish Diabetes Risk Score was implemented to assess the risk factors associated with diabetes. Results: $68.6 \%$ of the people were women. The $64.7 \%$ of the population were older than 45 years old. The $51.0 \%$ of the population had a score below 7 (low), 17.6\% between 7 and 11 (slightly high), $21.6 \%$ between 12 and 14 (moderate), 7.8\% higher than 14 and up to 20 (high), and $2.0 \%$ higher than 20 (very high). The average total score was 7.8 . The $51.0 \%$ of the population got a BMI less than $25 \mathrm{Kg} / \mathrm{m}^{2}, 66.7 \%$ do not performed daily physical activity, $47.1 \%$ do not eat fruit and vegetables, $94.1 \%$ do not had history of high levels of blood glucose, and $23.5 \%$ had a familiar history of diabetes mellitus with the first degree of consanguinity. In the association analysis, age, consumption of antihypertensive medicines and family history of diabetes mellitus had a considerable influence on the test final score. Conclusions: The Finnish Diabetes Risk Score is a useful tool to characterize the risk factors for type 2 diabetes mellitus.

Key words: Diabetes mellitus, glucose intolerance, metabolic diseases, obesity, Findrisk test.

Montes-Ochoa S, Serna-Arrieta K, Estrada-Ávila S, Guerra-López FR, Sánchez IP. Caracterización de los factores de riesgo de diabetes mellitus tipo 2 mediante el test de Findrisk en una población de 30 a 50 años de Medellín, Colombia. Medicina \& Laboratorio 2016; 22: 563-576.

L a diabetes mellitus comprende un grupo de trastornos metabólicos frecuentes que comparten el fenotipo de la hiperglucemia. Existen varios tipos de diabetes mellitus, resultado de una interacción compleja entre factores genéticos y ambientales. Los mecanismos descritos que contribuyen a la hiperglucemia incluyen la deficiencia de la secreción de la insulina, la disminución de la utilización de la glucosa o el aumento de la producción de esta, lo que ocasiona un daño progresivo de los órganos blanco que desencadena complicaciones con secuelas permanentes, incluso la muerte, en las personas que padecen esta enfermedad. Estas 
características convierten a la diabetes mellitus en una enfermedad de gran impacto no solo sobre el individuo que la padece, sino también en el sistema de salud, ya que por su condición crónica y las comorbilidades que tiene asociadas se incluye dentro de las enfermedades con más alto costo (1.427 USD por persona) [1,2].

La prevalencia de la diabetes mellitus tipo 2 a nivel mundial aumenta con mayor rapidez, al parecer por el incremento en estos últimos años en la frecuencia de la obesidad (más del $30 \%$ ), el síndrome metabólico (20\% al $35 \%$ ) y la disminución de actividad física [3]. De acuerdo a la Federación Internacional de Diabetes (FID) en el mundo hay 387.000 .000 de personas con diabetes mellitus [1]. Se calcula que en el 2014 fallecieron 4.900 .000 personas como consecuencia de esta enfermedad. En la región de América del Sur y Central, Colombia es el segundo país, después de Brasil, con mayor número de personas que padecen diabetes. La prevalencia de diabetes en Colombia pasó de ser en 2009 de 4,8\% para el grupo de personas entre 20 y 79 años de edad a ser en 2014 de 7,17\% (2.191 personas) en este mismo grupo de edad, con una mortalidad de 14.168 personas [2].

El test de Findrisk (del inglés, Finnish Diabetes Risk Score) es una herramienta que mediante ocho preguntas permite evaluar el riesgo de una persona de desarrollar diabetes mellitus tipo 2 en los próximos 10 años. Esta investigación tiene como objetivo desarrollar un estudio piloto para la caracterización de los factores de riesgo de diabetes mellitus tipo 2 mediante el test de Findrisk en un población de 30 a 50 años en la ciudad de Medellín, Colombia, que pueda ser utilizado en un futuro como una herramienta clave en la promoción y prevención de la diabetes mellitus tipo 2 en Colombia y el mundo.

\section{Materiales y métodos}

\section{Tipo de estudio y población}

Este es un estudio piloto, descriptivo, transversal, en el cual se incluyeron 51 personas mayores de 30 años y menores de 50 años de edad residentes en el área metropolitana de la ciudad de Medellín, Colombia. El número de personas a participar fue escogido por conveniencia, teniendo en cuenta que el test de Findrisk no ha sido validado en la población colombiana y que una de las finalidades del estudio era evaluar la practicidad del test para ser desarrollado por la población de estudio.

Los criterios de inclusión de los individuos del estudio, además del rango de edad establecido, fue la ausencia de un diagnóstico previo de diabetes mellitus tipo 1 o tipo 2. Los criterios de exclusión se establecieron para aquellas personas en condición de discapacidad, mujeres embarazadas, personas con enfermedades neurodegenerativas, diabetes mellitus tipo 1 o tipo 2, pacientes que no residieran en el área metropolitana de Medellín, Colombia, y no se encontraran dentro del rango de edad establecido. 
Las personas que accedieron a participar en el estudio lo hicieron de manera libre y voluntaria. Dicho consentimiento se expresó de manera verbal, al momento de diligenciar el test de Findrisk. Las variables evaluadas de las personas sometidas al test fueron: edad, índice de masa corporal (IMC), perímetro de cintura, actividad física, consumo de frutas y verduras, uso de medicamentos antihipertensivos y niveles previos de glucosa en sangre altos [4]. Para una mejor caracterización de la muestra de estudio se incluyeron también datos sociodemográficos.

\section{Evaluación de factores de riesgo de diabetes mellitus tipo 2}

Para la evaluación de los factores de riesgo de diabetes mellitus tipo 2 se empleó el test de Findrisk, respaldado por la Asociación Colombiana de Diabetes. Para una mejor caracterización se incluyeron datos de referencia de los individuos evaluados como el sexo, la ocupación y el estrato socioeconómico. Los resultados obtenidos en el test se evaluaron mediante un algoritmo usado por la Organización para la Excelencia de la Salud, revisado por la Dirección Científica de la Asociación Colombiana de Diabetes, en el cual se establece que las personas que clasifiquen con un puntaje en el test de Findrisk mayor que 13 poseen un mayor riesgo de desarrollar diabetes mellitus tipo 2 [5].

\section{Análisis estadístico}

Se realizó la distribución de frecuencias de los factores de riesgo en la población evaluada de diabetes mellitus tipo 2. Así mismo, se realizaron comparaciones entre las medias del puntaje final y las variables evaluadas usando la prueba de Mann Whitney y Tukey. El análisis de asociación se realizó usando una prueba de Fisher. Para el análisis estadístico se empleó el programa GraphPad Prisma 6,0 (GraphPad Software Inc., La Jolla, Estados Unidos).

\section{Resultados}

\section{Características sociodemográficas de la muestra de estudio}

Los resultados obtenidos mostraron que de las 51 personas que accedieron a participar en el estudio el $68,6 \%$ fueron mujeres y el 31,4\% hombres (véase figura 1). Teniendo en cuenta la subdivisión de los grupos etarios establecido por el test se encontró que el $64,7 \%$ de los individuos eran menores de 45 años y $35,3 \%$ se encontraban en el rango de edad entre los 45 y 54 años. No se encontraron personas dentro de los demás rangos de edad establecidos (véase figura 1 y tabla 1 ). De acuerdo al estrato socioeconómico el 45,0\% de la población de estudio era estrato 3, 29,4\% estrato $2,17,6 \%$ estrato 4, 3,9\% estrato 5 y el $3,9 \%$ restante estrato 1 . La distribución de la población, de acuerdo a su profesión u ocupación, fue muy variable, de tal manera que no se lograron establecer categorías. 


\section{Parámetros antropométricos asociados al riesgo de diabetes}

Dos de las variables evaluadas en el test de Findrisk fueron el IMC y el perímetro de cintura, establecidos como factores de riesgo para el desarrollo de diabetes mellitus tipo 2 [6]. En los individuos analizados se evidenció que el $51,0 \%$ presentaban un IMC menor que $25 \mathrm{~kg} / \mathrm{m}^{2}$, el $33,3 \%$ un IMC entre $25 \mathrm{~kg} / \mathrm{m}^{2}$ y $30 \mathrm{~kg} /$ $\mathrm{m}^{2}$ y el $15,6 \%$ un IMC mayor que $30 \mathrm{~kg} /$ $\mathrm{m}^{2}$ (peso normal, sobrepeso y obesidad, respectivamente), de acuerdo con la clasificación de la Organización Mundial de la Salud [7] (véase figura 1 y tabla 1 ).

El sobrepeso de acuerdo al IMC se presentó en el $75,0 \%$ de los hombres y el $14,3 \%$ de las mujeres y la obesidad en el $12,5 \%$ de los hombres y el $17,1 \%$ de

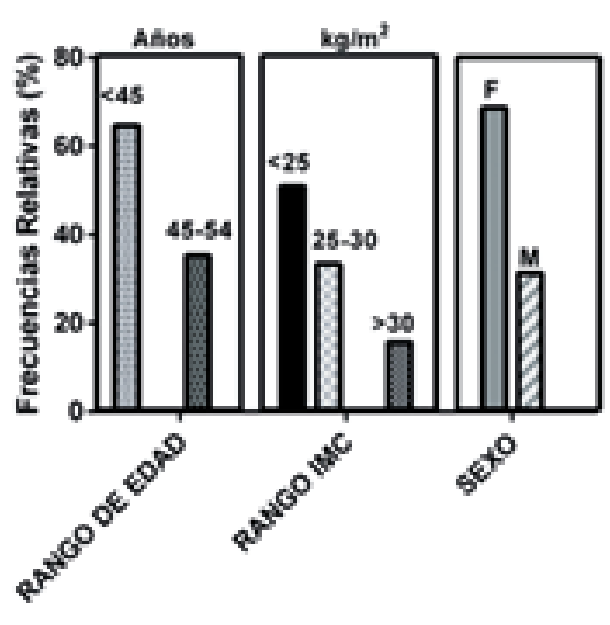

Figura 1. Distribución porcentual de la muestra de estudio según parámetros sociodemográficos y antropométricos. La figura describe el rango de frecuencias porcentuales para las variables rango de edad, índice de masa corporal (IMC) y sexo. La mayoría de la población se encuentra en un rango de edad menor que 45 años, con predominio de un valor de IMC menor que $25 \mathrm{~kg} / \mathrm{m}^{2}$ y principalmente de sexo femenino. las mujeres. El 31,3\% de los hombres presentaban un perímetro de cintura menor que $90 \mathrm{~cm}$, el $37,5 \%$ entre $90 \mathrm{~cm}$ y $98 \mathrm{~cm}$, y el $31,3 \%$ mayor que $98 \mathrm{~cm}$ (obesidad abdominal) [6]. En la población femenina el 31,4\% tenían un perímetro de cintura menor que $80 \mathrm{~cm}$, el $28,6 \%$ entre $80 \mathrm{~cm}$ y $88 \mathrm{~cm}$ y el $40,0 \%$ mayor que $88 \mathrm{~cm}$ (obesidad abdominal) [6] (véase tabla 1).

\section{Parámetros del estilo de vida asociados al riesgo de diabetes}

Entre las variables que definen el estilo de vida se evaluó en los individuos del estudio la actividad física (definida como 30 minutos de actividad cada día o cuatro horas semanales, en el trabajo o el tiempo libre) y el consumo de frutas y verduras, encontrando que el $66,7 \%$ de la población refirió no realizar actividad física, mientras el 33,3\% sí la realizaba, y el 47,1\% manifestó no consumir frutas y verduras, entretanto el $52,9 \%$ sí las consumía (véase tabla 1 ).

\section{Antecedentes personales de hipertensión arterial y nivelesde glucosa altos en sangre y antecedentes familiares de diabetes mellitus}

La alteración en el metabolismo de la glucosa hace parte de la definición del síndrome metabólico, en concomitancia con la hipertensión arterial y la obesidad [6]. En el presente estudio el $86,3 \%$ de la población no consumía medicamentos antihipertensivos, mientras que el $13,7 \%$ refirió que sí, representado en su totalidad por mujeres. Con respecto a los antecedentes personales de niveles de glucosa 
altos en sangre, el 5,9\% de la población de estudio los presentaba, todos estos casos mujeres (véase tabla 1 ).

\begin{tabular}{|c|c|c|c|}
\hline \multirow[t]{2}{*}{ Variables } & \multicolumn{3}{|c|}{ N. ${ }^{\circ}$ de individuos (\%) } \\
\hline & $\begin{array}{l}\text { Hombres } \\
(n=16 ; 31,4 \%)\end{array}$ & $\begin{array}{l}\text { Mujeres } \\
(n=35 ; 68,6 \%)\end{array}$ & $\begin{array}{l}\text { Total población } \\
(\mathrm{n}=51)\end{array}$ \\
\hline $\begin{array}{l}\text { Edad: } \\
\text { <45 años } \\
45-54 \text { años } \\
55-64 \text { años } \\
>64 \text { años }\end{array}$ & $\begin{array}{l}12(75,0) \\
4(25,0) \\
0(0) \\
0(0)\end{array}$ & $\begin{array}{l}21(60,0) \\
14(40,0) \\
0 \\
0\end{array}$ & $\begin{array}{l}33(64,7) \\
18(35,3) \\
0 \\
0\end{array}$ \\
\hline $\begin{array}{l}\text { Índice de masa corporal (IMC): } \\
<25 \mathrm{~kg} / \mathrm{m}^{2} \\
25-30 \mathrm{~kg} / \mathrm{m}^{2} \\
>30 \mathrm{~kg} / \mathrm{m}^{2}\end{array}$ & $\begin{array}{l}2(12,5) \\
12(75,0) \\
2(12,5)\end{array}$ & $\begin{array}{l}24(68,6) \\
5(14,3) \\
6(17,1)\end{array}$ & $\begin{array}{l}26(51,0) \\
17(33,3) \\
8(15,7)\end{array}$ \\
\hline $\begin{array}{l}\text { Perímetro de cintura: } \\
\text { Hombres: } \\
\text { < } 90 \mathrm{~cm} \\
90-98 \mathrm{~cm} \\
>98 \mathrm{~cm} \\
\text { Mujeres: } \\
<80 \mathrm{~cm} \\
80-88 \mathrm{~cm} \\
>88 \mathrm{~cm}\end{array}$ & $\begin{array}{l}5(31,3) \\
6(37,5) \\
5(31,3)\end{array}$ & $\begin{array}{l}11(31,4) \\
10(28,6) \\
14(40,0)\end{array}$ & \\
\hline $\begin{array}{l}\text { Obesidad abdominal (hombres } \\
>98 \mathrm{~cm} \text {, mujeres }>88 \mathrm{~cm} \text { ) } \\
\text { Presente } \\
\text { Ausente }\end{array}$ & & & $\begin{array}{l}20(39,2) \\
31(60,8)\end{array}$ \\
\hline $\begin{array}{l}\text { Actividad física: } \\
\text { No } \\
\text { Sí }\end{array}$ & $\begin{array}{l}10(62,5) \\
6(37,5)\end{array}$ & $\begin{array}{l}24(68,6) \\
11(31,4)\end{array}$ & $\begin{array}{l}34(66,7) \\
17(33,3)\end{array}$ \\
\hline $\begin{array}{l}\text { Frutas y verduras: } \\
\text { No } \\
\text { Sí }\end{array}$ & $\begin{array}{l}9(56,3) \\
7(43,8)\end{array}$ & $\begin{array}{l}15(42,9) \\
20(57,1)\end{array}$ & $\begin{array}{l}24(47,1) \\
27(52,9)\end{array}$ \\
\hline $\begin{array}{l}\text { Medicamentos para la } \\
\text { hipertensión arterial: } \\
\text { No } \\
\text { Sí }\end{array}$ & $\begin{array}{l}16(100) \\
0(0)\end{array}$ & $\begin{array}{l}28(80,0) \\
7(20,0)\end{array}$ & $\begin{array}{l}44(86,3) \\
7(13,7)\end{array}$ \\
\hline $\begin{array}{l}\text { Antecedentes de niveles de } \\
\text { glucosa altos: } \\
\text { No } \\
\text { Sí }\end{array}$ & $\begin{array}{l}16(100) \\
0(0)\end{array}$ & $\begin{array}{l}32(91,4) \\
3(8,6)\end{array}$ & $\begin{array}{l}48(94,1) \\
3(5,9)\end{array}$ \\
\hline $\begin{array}{l}\text { Antecedentes familiares de diabet } \\
\text { mellitus } \\
\text { No } \\
\text { Sí (segundo o tercer grado de } \\
\text { consanguinidad) } \\
\text { Sí (primer grado de consangui- } \\
\text { nidad) }\end{array}$ & $\begin{array}{l}11(68,8) \\
2(12,5)\end{array}$ & $\begin{array}{l}20(57,1) \\
6(17,1)\end{array}$ & $\begin{array}{l}31(60,8) \\
8(15,7)\end{array}$ \\
\hline
\end{tabular}

El antecedente familiar de la diabetes mellitus ha sido definido como un factor de riesgo para el desarrollo de la enfermedad [8]. En este estudio el $25,7 \%$ de las mujeres y el $18,7 \%$ de los hombres tienen antecedentes familiares de diabetes 
mellitus en primer grado de consanguinidad, lo cual representa el 23,5\% de la población total de estudio con este factor de riesgo. El 15,7\% de la población presentó antecedentes familiares de diabetes mellitus en segundo y tercer grado de consanguinidad, mientras que el $60,8 \%$ no manifestaron antecedentes familiares de diabetes mellitus (véase tabla 1 ).

\section{Análisis de relación entre el puntaje total del test de Findrisk y sus variables}

Con respecto al puntaje total final del test de Findrisk se encontró en el 51,0\% de la población un puntaje bajo (menor que 7 puntos), lo que indica que una de cada 100 personas desarrollará diabetes mellitus en un plazo de 10 años, el $17,6 \%$ un puntaje elevado levemente (7 a 11 puntos), equivalente a que una de cada 25 personas desarrollará diabetes mellitus en un plazo de 10 años, el $21,6 \%$ un puntaje moderado

\begin{tabular}{|lll|}
\hline \multicolumn{3}{|l}{ Tabla 2. Puntaje final test de Findrisk } \\
\hline Distribución puntaje final & $\begin{array}{l}\text { Frecuencia } \\
\text { absoluta }\end{array}$ & $\%$ \\
\hline Bajo $(<7)$ & 26 & 51,0 \\
\hline Elevado levemente (7-11) & 9 & 17,6 \\
\hline Moderado (12-14) & 11 & 21,6 \\
\hline Alto $(15-20)$ & 4 & 7,8 \\
\hline Muy alto $(>20)$ & 1 & 2,0 \\
\hline Total & 51 & 100 \\
\hline
\end{tabular}
(12 a 14 puntos), lo que indica que una de cada seis personas desarrollará diabetes mellitus en un plazo de 10 años, el 7,8\% un puntaje alto (15 a 20 puntos), indicativo que una de cada tres personas desarrollará diabetes mellitus en un plazo de 10 años y $2,0 \%$ un puntaje muy alto (mayor que 20 puntos), que equivale a que una de cada dos personas desarrollará diabetes mellitus en un plazo de 10 años (véase tabla 2).

La media del puntaje total en la población general fue de 7,8 , en los hombres de 7,06 y en las mujeres de 8,14 . El número de personas con un puntaje menor que 13 fue de 41 , de los cuales 27 eran mujeres y 14 hombres. Del total de personas 10 tuvieron un puntaje mayor o igual que 13, de los cuales ocho eran mujeres y 14 hombres. En la comparación de las medias del puntaje total en hombres y en mujeres se determinó que no había diferencia estadísticamente significativa (datos no mostrados).

La comparación del promedio del puntaje de las variables mostró diferencias estadísticamente significativas en los grupos de edad de menores de 45 años y el grupo de 45 a 54 años (valor $p=0,0001$ ). Así mismo, esta diferencia se observó entre los grupos sin antecedentes familiares de diabetes y el grupo con antecedentes en primer grado de consanguinidad (valor $p<0,0001$ ) y entre estos últimos y aquellos con antecedentes en segundo-tercer grado de consanguinidad (valor $p=0,0019$ ), entre los que no realizan actividad física y los que sí la realizan (valor $p=0,0004) \mathrm{y}$, entre los grupos que consumen y que no consumen medicamentos antihipertensivos (valor $p=0,0002$ ) (véase tabla 3 ).

El análisis de evaluación del riesgo relativo entre la edad menor que 45 años y entre 45 y 54 años con un puntaje menor que 13 y mayor o igual que 13 mostró 
que el segundo rango de edad representa un riesgo relativo de desarrollar diabetes mellitus de 3,7 veces más respecto al primer rango de edad $(p=0,0019)$. Así mismo, los antecedentes familiares de diabetes mellitus representan un riesgo relativo de 7,3 veces más respecto a las personas que no tienen estos antecedentes $(p=0,004)$ de desarrollar la enfermedad. La asociación entre el consumo de medicamentos antihipertensivos respecto a estos rangos del puntaje total mostró un riesgo relativo de 0,1 , que sin bien es bajo fue estadísticamente significativo $(p=0,02)$ (datos no mostrados).

\begin{tabular}{|c|c|c|c|}
\hline Variables & Media del puntaje & $\begin{array}{l}\text { Diferencia } \\
\text { entre las medias }\end{array}$ & Valor $p$ \\
\hline \multicolumn{4}{|l|}{ Edad } \\
\hline$<45$ & $5(n=33)$ & \multirow[t]{2}{*}{7} & \multirow[t]{2}{*}{0,0001} \\
\hline $45-54$ & $12(n=18)$ & & \\
\hline \multicolumn{4}{|l|}{ Índice de masa corporal (IMC) } \\
\hline$<25 \mathrm{~kg} / \mathrm{m}^{2}$ & $5,5(n=26)$ & \multirow{2}{*}{3,5} & \multirow{2}{*}{0,13} \\
\hline$>25 \mathrm{~kg} / \mathrm{m}^{2}$ & $9,0(n=25)$ & & \\
\hline \multicolumn{4}{|l|}{ Perímetro de cintura (PC) } \\
\hline $\begin{array}{l}\text { Obesidad abdominal: } \\
\text { Hombre }(P C>98 \mathrm{~cm})\end{array}$ & $12(n=5)$ & \multirow[t]{2}{*}{0} & \multirow[t]{2}{*}{0,8} \\
\hline $\begin{array}{l}\text { Obesidad abdominal: Mujeres (PC } \\
>88 \mathrm{~cm} \text { ) }\end{array}$ & $12(n=14)$ & & \\
\hline \multicolumn{4}{|l|}{ Actividad física } \\
\hline Sí & $4(n=33)$ & \multirow{2}{*}{6} & \multirow{2}{*}{0,0004} \\
\hline No & $10(n=18)$ & & \\
\hline \multicolumn{4}{|l|}{ Consumo de frutas y verduras } \\
\hline Sí & $6(n=27)$ & \multirow{2}{*}{3,5} & \multirow{2}{*}{0,08} \\
\hline No & $9.5(n=24)$ & & \\
\hline \multicolumn{4}{|l|}{ Medicamentos antihipertensivos } \\
\hline Sí & $13(n=7)$ & \multirow[t]{2}{*}{7} & \multirow{2}{*}{0,0002} \\
\hline No & $6(n=44)$ & & \\
\hline \multicolumn{4}{|l|}{$\begin{array}{l}\text { Antecedentes de glucosa elevada en } \\
\text { sangre }\end{array}$} \\
\hline Sí & $16(n=3)$ & \multirow{2}{*}{10} & \multirow{2}{*}{0,4} \\
\hline No & $6(n=48)$ & & \\
\hline \multicolumn{4}{|l|}{$\begin{array}{l}\text { Antecedentes familiares de diabetes } \\
\text { mellitus tipo } 2\end{array}$} \\
\hline No & $5,35(n=35)$ & $\begin{array}{l}\text { No antecedentes familiares fren- } \\
\text { te Sí antecedentes familiares en } \\
\text { segundo-tercer grado }\end{array}$ & 0,1650 \\
\hline $\begin{array}{l}\text { Sí (segundo y tercer grado de con- } \\
\text { sanguinidad) }\end{array}$ & $8(n=7)$ & $\begin{array}{l}\text { No antecedentes familiares fren- } \\
\text { te Sí antecedentes familiares en } \\
\text { primer grado }\end{array}$ & $<0,0001$ \\
\hline Sí (primer grado de consanguinidad) & $14(n=9)$ & $\begin{array}{l}\text { Si antecedentes familiares en } \\
\text { segundo-tercer grado frente } \\
\text { Sí antecedentes familiares en } \\
\text { primer grado }\end{array}$ & 0,0019 \\
\hline
\end{tabular}




\section{Discusión}

El test de Findrisk es una herramienta no invasiva y costo-efectiva para caracterizar los factores de riesgo relacionados con la diabetes mellitus tipo 2 [9-11]. Así mismo, en diversos estudios el test ha demostrado ser eficaz como instrumento de tamización para la detección de diabetes mellitus y de otros trastornos relacionados con el metabolismo de los carbohidratos [12]. En este estudio el 51,0\% de la población obtuvo un puntaje de riesgo de desarrollar diabetes tipo 2 en los próximos 10 años menor que 7 (bajo). La obesidad abdominal en las mujeres y la no actividad física fueron los factores de riesgo más prevalentes. La obesidad abdominal (definida por el perímetro de cintura) y la obesidad global (definida por el IMC) son uno de los factores de riesgo más importantes debido a que se relacionan con la resistencia a la insulina [13-15].

En Latinoamérica y el Caribe se estimó en 2010 una prevalencia de sobrepeso y obesidad de 36 por 1.000 habitantes, similar a lo encontrado en Suramérica para el mismo año, donde se reportó en 34 por 1.000 habitantes [16]. En Colombia la obesidad en las personas entre los 18 y 64 años ha tendido a incrementar, con una prevalencia para $201020 \%$ mayor que para 2005, pasando de 13,70 a 16,50 casos por cada 100 personas [17].

En la población colombiana la obesidad es mayor en las mujeres respecto a los hombres en un $75 \%$, con una diferencia absoluta de 8,6 mujeres obesas más por cada 100 personas. Así mismo, la obesidad presenta una mayor frecuencia (19\%) en el área urbana que en la rural, con una diferencia absoluta de 2,8 obesos más por cada 100 personas, y entre las personas que se autorreconocen como afrocolombianos ( $11 \%$ más) comparado con el resto de la población, para una diferencia absoluta de 1,8 obesos más [17].

Un aspecto socioeconómico que se ha mostrado influyente en el desarrollo de obesidad es el nivel educativo. En Colombia se ha evidenciado que la obesidad es un $26 \%$ mayor en las personas sin ningún nivel educativo respecto a los de grado superior, para una diferencia absoluta de 3,6 personas obesas más. En cuanto a los departamentos donde mayor obesidad se reportó entre 2005 y 2010, en personas con edades entre los 18 y 64 años, fueron San Andrés, Guaviare, Caquetá, Tolima, Guainía, Chocó, Casanare y Arauca. Específicamente, en el departamento de Antioquia el porcentaje de obesidad y sobrepeso fue del $15 \%$ [17].

En el presente estudio el rango de edad mayor de 45 años, el consumo de medicamentos antihipertensivos y los antecedentes familiares de diabetes mellitus fueron los factores de riesgo que tuvieron asociación con el puntaje total del test de Findrisk. En Estados Unidos, en 2011, de acuerdo a los CDC el 63\% de los casos incidentes de diabetes mellitus en adultos fueron diagnosticados entre los $40 \mathrm{y}$ los 69 años [18]. Entretanto, en el quinquenio 2009-2013, en Colombia el 92,2\% 
de las personas diagnosticadas con diabetes mellitus fueron mayores de 45 años [17]. Estos datos son concordantes con lo referenciado en la literatura científica, donde se sugiere que en el páncreas ocurren cambios degenerativos con la edad, que producen como consecuencia alteración en el metabolismo de la glucosa [19].

La hipertensión arterial es una enfermedad que a menudo se presenta concomitante con la diabetes mellitus, aumentando significativamente su mortalidad y morbilidad [20]. La diabetes mellitus causa una disfunción en el endotelio de los vasos sanguíneos, lo que se cree está relacionado con la posterior aparición de la hipertensión arterial [21]. La disfunción endotelial es un fenómeno temprano en la diabetes mellitus, existen evidencias de que los marcadores de disfunción endotelial están elevados en los pacientes diabéticos tipo 2 , años antes de que la enfermedad se manifieste clínicamente $[21,22]$.

En Colombia, de acuerdo al Análisis de Situación de Salud (ASIS) del quinquenio 2009-2013, la prevalencia de hipertensión arterial en las personas afiliadas al Sistema General de Seguridad Social en Salud (SGSSS) fue de 6,4 personas por cada 100 afiliados. El 91,5\% de las personas diagnosticadas con hipertensión arterial eran mayores de 40 años [17]. Estos hallazgos evidencian que en nuestro país la prevalencia, tanto de la hipertensión arterial como de la diabetes mellitus, es elevada y con valores que son equiparables entre sí a partir de los 40 años, lo cual podría sugerir una asociación entre ambas entidades.

En la última década diversas investigaciones han demostrado que existe asociación entre los antecedentes familiares de diabetes mellitus y la presencia de la enfermedad. En las personas con estos antecedentes el riesgo de padecer diabetes mellitus se incrementa de dos a seis veces cuando se presenta en familiares en primer grado de consanguinidad [23-25], lo que sugiere un patrón hereditario. En este estudio el riesgo relativo, encontrado en el análisis de asociación, de los antecedentes familiares de diabetes mellitus en primer grado de consanguinidad respecto al puntaje final del test de Findrisk fue de 7,3 .

En 2007, en la revista Nature, se publicó una investigación en la que mediante el estudio del genoma humano se establecieron cuatro genes, TCF7L2, HHEX, EXT2 y SLC30A8, que podrían explicar la heredabilidad de la diabetes mellitus tipo 2 hasta en un $70 \%$ de los casos [26]. Así mismo, en años posteriores se han desarrollado estudios con el fin de establecer con certeza cuál es la relevancia de estos genes en la patogenia de la enfermedad $[27,28]$.

La validación externa del test de Findrisk se ha realizado en 11 países: Alemania, Reino Unido, Bulgaria, China, Kuwait, Taiwán, Filipinas, Italia, España, Estados Unidos y Grecia [10]. Hasta el momento en Colombia aún no se ha llevado a cabo la validación de una herramienta para realizar la caracterización o el tamizaje de la diabetes mellitus tipo 2; en la actualidad el referente es el estudio DEMOJUAN 
(del inglés, DEMOnstration area for primary prevention of type 2 diabetes, JUAN Mina and Soledad, Barranquilla, Colombia) realizado por la Subdirección de Enfermedades Crónicas No Transmisibles, del Ministerio de Salud y Protección Social en la ciudad de Barranquilla, Colombia. Este estudio demostró que los factores de riesgo más prevalentes de diabetes mellitus tipo 2 son la obesidad y el sobrepeso, combinado con la baja actividad física [5], que coinciden con lo hallado en el presente estudio, en el que los factores de riesgo encontrados fueron: obesidad abdominal, no consumo de frutas y verduras y no actividad física.

El estudio DEMOJUAN es la plataforma por medio de la cual se están iniciando estrategias para la validación del test de Findrisk en Colombia. El instrumento a validar, denominado COLDRISC, posee cuatro variables: edad, perímetro abdominal, uso de medicamentos antihipertensivos e historia familiar de diabetes mellitus (padres o hermanos). Sin embargo, este test aún no está disponible para su implementación oficial en las entidades de salud en nuestro país [29].

En la presente investigación se encontró que el $49,0 \%$ de la población tuvo un puntaje del test de Findrisk mayor que 7. Diversos estudios aleatorios han encontrado que con un puntaje mayor o igual a 9 el test posee una elevada sensibilidad (78\%) y especificidad ( $48 \%$ al $77 \%$ ) para hacer el diagnóstico de diabetes y prediabetes $[4,9]$. En el estudio de validación del test de Findrisk, realizado en Finlandia entre 1980 y 1992, se hizo un seguimiento de 10 años a los participantes, encontrando que la incidencia de diabetes durante el seguimiento fue del $4,1 \%$. Con un punto de corte mayor o igual que 9 el test tuvo una sensibilidad del $81 \%$ y una especificidad del $76 \%$ para el diagnóstico de diabetes, identificando más del $70 \%$ de los casos incidentes [4].

De igual modo, en la evaluación de un modelo de prueba, basado en el test de Findrisk y la hemoglobina glicosilada (HbA1c), para el tamizaje de diabetes meIlitus y prediabetes, realizado en Estados Unidos y en el que se incluyeron 3.886 personas, se encontró que la incidencia de diabetes mellitus fue del 5,3\%. La sensibilidad y especificidad del test de Findrisk solo (punto de corte mayor o igual a 9), para diagnosticar diabetes mellitus, fue del $79,1 \%$ y $48,6 \%$, y para prediabetes de $60,2 \%$ y del $61,4 \%$, respectivamente. Un hallazgo de gran relevancia de dicho trabajo fue el incremento de la sensibilidad y la especificidad del test cuando se combinó con el parámetro bioquímico de hemoglobina glicosilada (HbA1c), las cuales fueron del $84,2 \%$ y el $48,4 \%$ para el diagnóstico de diabetes y del $74,2 \%$ y el 53,0\% para el diagnóstico de prediabetes, respectivamente [9]. Es importante resaltar que los valores de sensibilidad y especificidad del test se mantienen elevados aun usando puntos de corte más altos [30-32].

Por otra parte, se ha evaluado la capacidad del test para predecir la probabilidad de desarrollar diabetes mellitus. Como se mencionó anteriormente, el estudio inicial de validación en Finlandia, mediante el seguimiento a la población, esta- 
bleció, de acuerdo al puntaje final del test, la probabilidad de desarrollar diabetes mellitus a 10 años [4]. En la actualidad, para disminuir el impacto de la diabetes mellitus en la población mundial se ha venido ampliando este enfoque predictivo $y$, en este sentido, los hallazgos en la literatura científica revelan que en España se está desarrollando un estudio de este tipo, el cual se espera concluya en el año 2018, cuyo objetivo es implementar un modelo predictivo de diabetes mellitus adaptado a su país [10].

El presente estudio, por ser de tipo piloto y manejar una población reducida, hace difícil poder evaluar con exactitud la asociación entre las variables y el puntaje final, presentando un margen de error elevado. Sin embargo, los resultados obtenidos concuerdan con lo encontrado en la literatura científica. Para una mejor caracterización y análisis de asociación sería adecuado manejar una muestra representativa de la población de la ciudad de Medellín, Colombia, con criterios de exclusión más estrictos, de manera que se pueda hacer un análisis objetivo de la capacidad del test de Findrisk para caracterizar los factores de riesgo de diabetes mellitus tipo 2. Así mismo, cabría la realización de pruebas bioquímicas, como la prueba de tolerancia oral a la glucosa, que es la prueba más sensible para el diagnóstico de diabetes mellitus [33], con el fin de establecer con qué exactitud el test detecta realmente a aquellas personas con la enfermedad.

\section{Conclusiones}

Podemos concluir que es de gran aporte a nuestro país que se esté desarrollando una herramienta que permita realizar el diagnóstico oportuno y la prevención de la diabetes por parte de la Subdirección de Enfermedades Crónicas No Transmisibles del Ministerio de Salud y Protección Social, la cual sea costo-efectiva y de fácil implementación en el sistema de atención primaria en salud, y disminuya el gasto que lleva consigo la atención de una enfermedad crónica cada día más prevalente.

\section{Agradecimientos}

A Noel Barengo de la Subdirección del Departamento de Enfermedades Crónicas No Transmisibles del Ministerio de Salud y Protección Social por su aporte de la literatura que se está adelantando en el país y que al momento de la realización de esta revisión no se tenía conocimiento de su publicación en el tema. De igual manera, a los profesionales de la Corporación Universitaria Remington: Isaura Sánchez por la asesoría metodológica, Foris Guerra López por la asesoría temática y Rubén Mejía, Biólogo, Coordinador de Ciencias Básicas, y María Elena Villegas, vicerrectora administrativa y financiera, por facilitar las condiciones locativas para la realización del estudio. 


\section{Bibliografía}

1. International Diabetes Federation. IDF Diabetes Atlas (ed 6ta ed). Bruselas, Bélgica; 2013.

2. American Diabetes Association. Información básica de la diabetes. 2015. Disponible: http://www.diabetes.org/es/informacion-basica-de-la-diabetes/. Consultado: dic 2015.

3. Aschner P. Epidemiología de la diabetes en Colombia. Av Diabetol 2010; 26: 95-100.

4. Lindstrom J, Tuomilehto $\mathbf{J}$. The diabetes risk score: a practical tool to predict type 2 diabetes risk. Diabetes Care 2003; 26: 725731.

5. Barengo NC, Acosta T, Arrieta A, Ricaurte C, Mayor D, Tuomilehto JO. Screening for people with glucose metabolism disorders within the framework of the DEMOJUAN project (DEMOnstration area for primary prevention of type 2 diabetes, JUAN Mina and Barranquilla, Colombia). Diabetes Metab Res Rev 2013.

6. Asociación Latinoamericana de Diabetes (ALAD). Guías ALAD 2006 de diagnóstico, control y tratamiento de la Diabetes Mellitus Tipo 2. Washington D.C., Estados Unidos: Organización Panamericana de la Salud; 2008.

7. Organización Mundial de la Salud. Global Database on Body Mass Index: BMI classification. 2015. Disponible: http://apps.who. int/bmi/index.jsp?introPage=intro_3.html. Consultado: dic 2015.

8. American Diabetes Association. 2015 American Diabetes Association (ADA) Diabetes Guidelines: Summary Recommendations from NDEI. 2015. Disponible: http://www. ndei.org/ADA-2013-Guidelines-Criteria-Diabetes-Diagnosis.aspx.html.

9. Zhang $\mathbf{Y}$, Hu G, Zhang L, Mayo R, Chen L. A novel testing model for opportunistic screening of pre-diabetes and diabetes among U.S. adults. PLoS One 2015; 10: e0120382.

10. Salinero-Fort MA, de Burgos-Lunar $\mathbf{C}_{\boldsymbol{r}}$ Mostaza Prieto J, Lahoz Rallo C, Abanades-Herranz JC, Gomez-Campelo P, et al. Validating prediction scales of type 2 diabetes mellitus in Spain: the SPREDIA- 2 population-based prospective cohort study protocol. BMJ Open 2015; 5: e007195.

11. de Oliveira Araujo L, e Silva ES, de Oliveira Mariano J, Moreira RC, Prezotto KH, Fernandes CA, et al. [Risk of developing diabetes mellitus in primary care health users: a cross-sectional study]. Rev Gaucha Enferm 2015; 36: 77-83.

12. Hellgren $\mathbf{M I}$, Petzold $\mathbf{M}$, Bjorkelund $\mathbf{C}_{\boldsymbol{r}}$ Wedel H, Jansson PA, Lindblad U. Feasibil- ity of the FINDRISC questionnaire to identify individuals with impaired glucose tolerance in Swedish primary care. A cross-sectional population-based study. Diabet Med 2012; 29: 1501-1505.

13. Tangvarasittichai S. Oxidative stress, insulin resistance, dyslipidemia and type 2 diabetes mellitus. World J Diabetes 2015; 6: 456480 .

14. Abbasi F, Brown BW, Jr., Lamendola C, McLaughlin T, Reaven GM. Relationship between obesity, insulin resistance, and coronary heart disease risk. J Am Coll Cardiol 2002; 40: 937-943.

15. Vincent HK, Taylor AG. Biomarkers and potential mechanisms of obesity-induced oxidant stress in humans. Int J Obes (Lond) 2006; 30: 400-418.

16. Food and Agriculture Organization of United Nations. The state of food and agriculture. Roma, Italia; 2013.

17. República de Colombia, Ministerio de Salud y Protección Social. Análisis de Situación de Salud COLOMBIA 2014. Bogotá D.C., Colombia: Imprenta Nacional de Colombia; 2014.

18. Centers for Disease Control and Prevention. Distribution of Age at Diagnosis of Diabetes Among Adult Incident Cases Aged 18-79 Years, United States, 2011. Atlanta, Estados Unidos. 2015. Disponible: http:// www.cdc.gov/diabetes/statistics/age/fig1. htm. Consultado: dic 2015.

19. Bacos K, Gillberg L, Volkov P, Olsson AH, Hansen T, Pedersen $\mathbf{O}$, et al. Blood-based biomarkers of age-associated epigenetic changes in human islets associate with insulin secretion and diabetes. Nat Commun 2016; 7: 11089 .

20. Sowers JR, Epstein M, Frohlich ED. Diabetes, hypertension, and cardiovascular disease: an update. Hypertension 2001; 37: 1053-1059.

21. Cruz Hernández J, Licea Puig ME, Hernández García P, Yanes Quesada M, Salvato Dueñas A. Disfunción endotelial y diabetes mellitus. Rev Cubana Endocrinol 2012; 23: $166-185$.

22. Odegaard AO, Jacobs DR, Jr., Sanchez OA, Goff DC, Jr., Reiner AP, Gross MD. Oxidative stress, inflammation, endothelial dysfunction and incidence of type 2 diabetes. Cardiovasc Diabetol 2016; 15: 51.

23. Harrison TA, Hindorff LA, Kim H, Wines RC, Bowen DJ, McGrath BB, et al. Family history of diabetes as a potential public health 
tool. Am J Prev Med 2003; 24: 152-159.

24. Meigs JB, Cupples LA, Wilson PW. Parental transmission of type 2 diabetes: the Framingham Offspring Study. Diabetes 2000; 49: 2201-2207.

25. Valdez R, Yoon PW, Liu T, Khoury MJ. Family history and prevalence of diabetes in the U.S. population: the 6-year results from the National Health and Nutrition Examination Survey (1999-2004). Diabetes Care 2007; 30: 2517-2522.

26. Sladek R, Rocheleau G, Rung J, Dina C, Shen L, Serre D, et al. A genome-wide association study identifies novel risk loci for type 2 diabetes. Nature 2007; 445: 881-885.

27. McCarthy MI, Zeggini E. Genome-wide association studies in type 2 diabetes. Curr Diab Rep 2009; 9: 164-171.

28. Wheeler E, Barroso I. Genome-wide association studies and type 2 diabetes. Brief Funct Genomics 2011; 10: 52-60.

29. Barengo N, Pombo L. Sesión No. 3. ODC: Implementación de Coldrisc, modelo de atención. $24^{\circ}$ Foro internacional OES, Garantizar la calidad en salud: Un sueño posible. Bogotá D.C., Colombia: Organización para la Excelencia en Salud (OES); 2015.
30. Tankova T, Chakarova N, Atanassova I, Dakovska L. Evaluation of the Finnish Diabetes Risk Score as a screening tool for impaired fasting glucose, impaired glucose tolerance and undetected diabetes. Diabetes Res Clin Pract 2011; 92: 46-52.

31. Commonwealth of Australia, Diabetes Australia Guideline Development Consortium. National Evidence Based Guideline for Case Detection and Diagnosis of Type 2 Diabetes. 2009. Disponible: http://static. diabetesaustralia.com.au/s/fileassets/diabetes-australia/af2389ea-8f61-4c54-82d677ab07f03597.pdf. Consultado: dic 2015.

32. Gomez-Arbelaez D, Alvarado-Jurado L, Ayala-Castillo M, Forero-Naranjo L, Camacho PA, Lopez-Jaramillo P. Evaluation of the Finnish Diabetes Risk Score to predict type 2 diabetes mellitus in a Colombian population: A longitudinal observational study. World J Diabetes 2015; 6: 1337-1344.

33. Reinauer H, Home PD, Kanagasabapathy AS, Heuck C. Diagnóstico y Monitorización de la Diabetes Mellitus desde el Laboratorio. Madrid, España: Organización Mundial de la Salud, Momento Médico Iberoamericana; 2005.

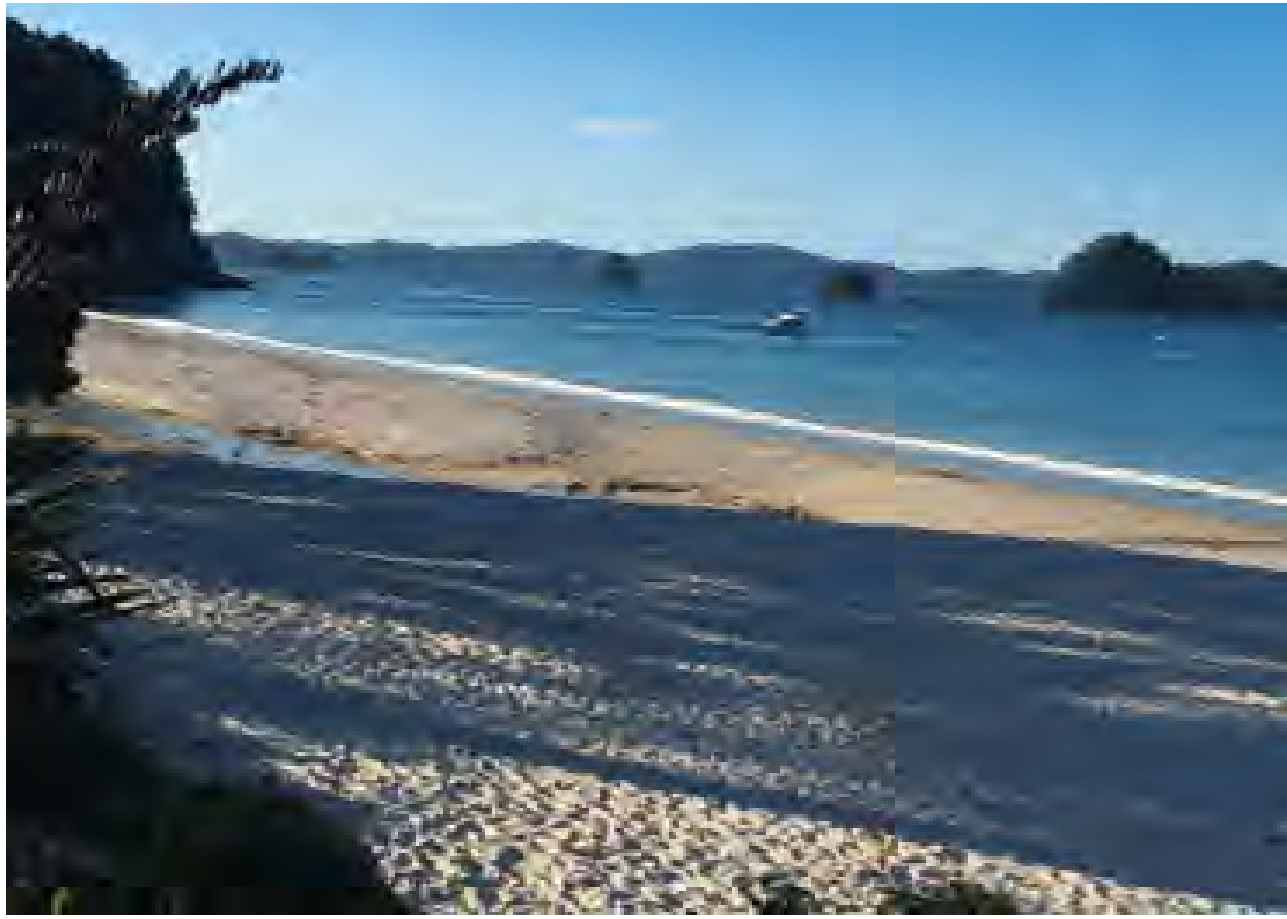

Hahei, Whitianga \& Rotorua.

New Zealand.

Carlos Alberto Lozano M. carlos.lozano@iatm.com.co 